International Journal of Number Theory

Vol. 11, No. 8 (2015) 2513-2520

(C) World Scientific Publishing Company

DOI: $10.1142 /$ S1793042115990018

\title{
Author index (Volume 11)
}

A

Alaca, S.., see Alaca, A.

Adam, D. \& Chabert, J.-L., On Bhargava's factorials of the set of twin primes in $\mathbb{Z}$ and in $\mathbb{F}_{q}[T]$

Adhikari, S. D. \&

Mazumdar, E., The polynomial method in the study of zero-sum theorems

Adiceam, F., Rational approximation and arithmetic progressions

Ahlgren, S. \& Kim, B., Dissections of a

"strange" function

Ahmed, Z. \& Baruah, N. D., New congruences for Andrews' singular overpartitions

Ahmed, Z. \& Baruah, N.

D., Parity results for broken 5-diamond,

7-diamond and 11-diamond partitions

Alaca, A., Alaca, Ş. \& Aygin, Z. S., Fourier coefficients of a class of eta quotients of weight 2

Alaca, A. \& Kesicioğlu, M. N., Representations by octonary quadratic forms with coefficients 1, 3

or 9

Alba González, J. J.,

Berrizbeitia, P. \& Luca,

F., On the formula

$F_{p}=u^{2}+p v^{2}$

Alkan, E., Series

representing

transcendental numbers

that are not $U$-numbers
1 (2015) 185

8 (2015) 2381

6 (2015) 1941

5 (2015) 1451

$2(2015) 451$

5 (2015) 1557

7 (2015) 2247

$2(2015) 527$

8 (2015) 2353

3 (2015) 869
8 (2015) 2381
Andrews, G. E., Singular overpartitions

5 (2015) 1523

Aoki, M. \& Kishi, Y., On systems of fundamental units of certain quartic fields

7 (2015) 2019

Aygin, Z. S., see Alaca, A.

Azizi, A., Zekhnini, A.,

Taous, Md. \& Mayer, D. C., Principalization of 2-class groups of type $(2,2,2)$ of biquadratic fields $\mathbb{Q}\left(\sqrt{p_{1} p_{2} q}, \sqrt{-1}\right)$

8 (2015) 2381

4 (2015) 1177

B

Baba, S. \& Granath, H., Quaternionic modular forms and exceptional sets of hypergeometric functions

2 (2015) 631

Backhausz, T. \& Zábrádi, G., Algebraic functional equations and completely faithful Selmer groups

4 (2015) 1233

Balasubramanian, R. \& Giri, S., On additive representation functions

Bănescu, M. \& Popa, D., Asymptotic evaluations for some double sums in number theory

Baoulina, I. N., Generalizations of the Markoff-Hurwitz equations over residue class rings

Barman, R. \& Saikia, N., Certain transformations for hypergeometric series in the $p$-adic setting

Baruah, N. D. \& Das, K., Parity results for 7-regular and 23-regular partitions
7 (2015) 2073

3 (2015) 909

4 (2015) 1165

7 (2015) 2221 
Baruah, N. D., see Ahmed, Z.

Baruah, N. D., see Ahmed, Z.

Benatar, J., The existence of small prime gaps in subsets of the integers

Beneish, L. \& Larson, H., Traces of singular values of Hauptmoduln

Bennett, M. A., Chen, I., Dahmen, S. R. \& Yazdani, S., Generalized Fermat equations: A miscellany

Berndt, B. C. \& Kim, S., Logarithmic means and double series of Bessel functions

Berrizbeitia, P., see Alba González, J. J.

Birbrair, L., Gomes, M. \& Pereira, W., Resonance sequences and recoverability

Biswas, S., Groups of components of Néron models of Jacobians and Brauer groups

Borovoi, M., Homogeneous spaces of Hilbert type

Bravo, J. J., Das, P., Guzmán, S. \& Laishram, S., Powers in products of terms of Pell's and Pell-Lucas sequences

Broderick, R. \& Kleinbock, D., Dimension estimates for sets of uniformly badly approximable systems of linear forms

Bugeaud, Y. \& Pejković, T., Quadratic approximation in $\mathbb{Q}_{p}$

C

Cai, T., Chen, D. \& Zhang, Y., Perfect numbers and Fibonacci primes (I)

Cégielski, P., Grigorieff, S. \& Guessarian, I., Newton representation of functions over natural integers having integral difference ratios
2 (2015) 527

7 (2015) 2247

3 (2015) 801

3 (2015) 1027

1 (2015) 1

5 (2015) 1535

1 (2015) 185

2 (2015) 495

2 (2015) 621

2 (2015) 397

4 (2015) 1259

7 (2015) 2037

$1(2015) 193$

1 (2015) 159

7 (2015) 2109
Chabert, J.-L., see

Adam, D.

6 (2015) 1941

Chan, T. H., Factors of almost squares and lattice points on circles

Chen, D., see Cai, T.

Chen, H. B., Wang, X. H. \& Wen, Z. X., Multifractal analysis on the level sets described by moving averages 7 (2015) 2175

Chen, I., see Bennett, M. A.

1 (2015) 1

Chen, S.-C., Hirschhorn, M. D. \& Sellers, J. A., Arithmetic properties of Andrews' singular overpartitions

Chen, W. W. L., Davenport's theorem in geometric discrepancy theory

5 (2015) 1701

1 (2015) 159

5 (2015) 1463

5 (2015) 1437

Chen, Y., Dunn, N., Hewett, C. \& Silas, S., Dedekind sums $s(a, b)$ and inversions modulo $b$

8 (2015) 2325

Chou, W.-S. \& Hsu, L. C., An extension of Fleck-type Möbius function and inversion

6 (2015) 1807

Choudhry, A., Sextuples of integers whose sums in pairs are squares

2 (2015) 543

Chow, S. \& Ghitza, A., Distinguishing newforms

3 (2015) 893

Chua, L., Gunby, B., Park, S. \& Yuan, A., Proof of a conjecture of Guy on class numbers

4 (2015) 1345

Cui, S.-P., Gu, W. X. \& Ma, Z. S., Congruences for partitions with odd parts distinct modulo 5

7 (2015) 2151

D

Dahmen, S. R., see Bennett, M. A.

1 (2015) 1

Daileda, R. \& Jones, N., On primitivity of Dirichlet characters

6 (2015) 1913

Das, K., see Baruah, N. D. Das, P., see Bravo, J. J. 7 (2015) 2221

4 (2015) 1259

Das, S. \& Meher, J., On quasimodular eigenforms

3 (2015) 835 
Defant, C., On ranges of variants of the divisor functions that are dense

Dickson, M., Local spectral equidistribution for degree two Siegel modular forms in level and weight aspects

Dudek, A. W., On the Riemann hypothesis and the difference between primes

Dunn, N., see Chen, Y.

E

Elliott, P. D. T. A., Additive and multiplicative functions from Nottingham to Urbana-Champaign

Evans, R. J. \& Williams, K. S., Quartic residuacity and the quadratic character of certain quadratic

irrationalities

F

Faye, B. \& Luca, F., On the equation $\varphi\left(X^{m}-1\right)=X^{n}-1$

Fernández, J. L. \& Fernández, P., Asymptotic normality and greatest common divisors

Fernández, P., see Fernández, J. L.

Filaseta, M., Graham, S. \& Trifonov, O., Starting with gaps between $k$-free numbers

Freiberg, T. \& Pomerance, C., A note on square totients

Freitas, N. \& Tsaknias, P., Criteria for $p$-ordinarity of families of elliptic curves over infinitely many number fields

Fu, R., see Yang, H.

G

Gao, W. D., Zhao, P. P. \& Zhuang, J. J., Zero-sum subsequences of distinct lengths
6 (2015) 1905

2 (2015) 341

3 (2015) 771

8 (2015) 2325

5 (2015) 1357

8 (2015) 2487

5 (2015) 1691

1 (2015) 89

1 (2015) 89

5 (2015) 1411

8 (2015) 2265

1 (2015) 81

4 (2015) 1107

7 (2015) 2141
Ghitza, A., see Chow, S.

Gil, B. K., Han, J.-W.,

Kim, T. H., Koo, R. H., Lee, B. W., Lee, J.,

Nam, K. S., Park, H. W. \& Park, P.-S., Frobenius numbers of Pythagorean triples

Gil, B. K., Han, J.-W., Kim, T. H., Koo, R. H., Lee, B. W., Lee, J., Nam, K. S., Park, H. W. \& Park, P.-S., Acknowledgment of Priority — Frobenius numbers of Pythagorean triples

3 (2015) 893

2 (2015) 613

3 (2015) 1061

4 (2015) 1165

Balasubramanian, R.

Girstmair, K., On the fractional parts of Dedekind sums

1 (2015) 29

Gomes, M., see Birbrair, L.

2 (2015) 495

Graham, S., see Filaseta, M.

Granath, H., see Baba, S.

Grigorieff, S., see Cégielski, P.

Gu, W. X., see Cui, S.-P.

Guerreiro, J., An orthogonality relation for a thin family of $G L(3)$ Maass forms

8 (2015) 2277

Guessarian, I., see Cégielski, $\mathrm{P}$.

Gunby, B., see Chua, L.

Guo, L., Lei, P. \& Zhao, J., Families of weighted sum formulas for multiple zeta values

Guo, V. J. W., Some congruences related to the q-Fermat quotients

Guzmán, S., see Bravo, J. J.

5 (2015) 1411

2 (2015) 631

7 (2015) 2109

7 (2015) 2151

7 (2015) 2109

4 (2015) 1345

3 (2015) 997

3 (2015) 1049

4 (2015) 1259

H

Hajli, M., On an arithmetic inequality on $\mathbb{P}_{\mathbb{Q}}^{1}$

Han, G.-N. \& Wu, W., Evaluations of the Hankel determinants of a Thue-Morse-like sequence
4 (2015) 1227 
Han, J.-W., see Gil, B. K.

Han, J.-W., see Gil, B. K.

Harrington, J., Two questions concerning covering systems

Hewett, C., see Chen, Y.

Hindes, W., Rational points on certain families of symmetric equations

Hirschhorn, M. D., see Chen, S.-C.

Hong, S., see Qin, X.

Hsu, L. C., see Chou, W.-S.

Hyodo, F., A formal power series of a Hecke ring associated with the Heisenberg Lie algebra over $\mathbb{Z}_{p}$

\section{J}

Jacobson, Jr., M. J., Lee, Y., Scheidler, R. \& Williams, H. C., Construction of all cubic function fields of a given square-free discriminant

Jennings-Shaffer, C., Rank and crank moments for partitions without repeated odd parts

Jones, N., see Daileda, R.

K

Karaatli, O., see Keskin, R.

Kesicioğlu, M. N., see Alaca, A.

Keskin, R. \& Karaatli, O., Generalized Fibonacci and Lucas numbers of the form $5 x^{2}$

Kettlestrings, D. \& Thunder, J. L., Counting points of given height that generate a quadratic extension of a function field

Kim, B., see Ahlgren, S.

Kim, S., see Berndt, B. C. Kim, T. H., see Gil, B. K. Kim, T. H., see Gil, B. K. Kishi, Y., see Aoki, M.

Kleinbock, D., see

Broderick, R.
2 (2015) 613

3 (2015) 1061

6 (2015) 1739

8 (2015) 2325

6 (2015) 1821

5 (2015) 1463

2 (2015) 437

6 (2015) 1807

8 (2015) 2305

6 (2015) 1839

3 (2015) 683

6 (2015) 1913

3 (2015) 931

8 (2015) 2353

3 (2015) 931

2 (2015) 569

5 (2015) 1557

5 (2015) 1535

2 (2015) 613

3 (2015) 1061

7 (2015) 2019

7 (2015) 2037
Kolitsch, L. W., Another approach to the Truncated Pentagonal Number Theorem

5 (2015) 1563

Koo, R. H., see Gil, B. K.

Koo, R. H., see Gil, B. K.

Koukoulopoulos, D.,

Primes in short arithmetic progressions

Kozek, M., Luca, F., Pollack, P. \& Pomerance, C., Harmonious pairs

2 (2015) 613

3 (2015) 1061

5 (2015) 1499

5 (2015) 1633

Kraus, A., Équation de Fermat et nombres premiers inertes

8 (2015) 2341

Kuan, Y.-L., A function field analogue of Romanoff's theorem

7 (2015) 2161

L

Laishram, S., see Bravo, J. J.

4 (2015) 1259

Larson, H., see Beneish, L.

Le, G., Schmidt's subspace theorem for moving hypersurface targets

3 (2015) 1027

Lee, B. W., see Gil, B. K.

Lee, B. W., see Gil, B. K.

Lee, J., see Gil, B. K.

Lee, J., see Gil, B. K.

Lee, Y., see Jacobson, Jr., M. J.

Lei, P., see Guo, L.

Leonardis, A., Cyclotomic approximation lattices

Li, B. \& Mance, B., Number theoretic applications of a class of Cantor series fractal functions, II

1 (2015) 139

2 (2015) 613

3 (2015) 1061

2 (2015) 613

3 (2015) 1061

6 (2015) 1839

3 (2015) 997

2 (2015) 557

Li, X.-J., On the explicit formula related to Riemann's zeta-function

Lim, M. F., A remark on the $\mathfrak{M}_{H}(G)$-conjecture and Akashi series

1 (2015) 269

Lim, M. F., On the pseudo-nullity of the dual fine Selmer groups

2 (2015) 407

8 (2015) 2451

Liu, Z.-G. \& Zeng, J., Two 7 (2015) 2055 expansion formulas involving the Rogers-Szegö polynomials with applications

2 (2015) 507 
Lo, C. W. K. \& Marcolli, M., $\mathbb{F}_{\zeta}$-geometry, Tate motives, and the Habiro ring

Louboutin, S. R., Real zeros of Dedekind zeta functions

Luca, F., see Alba González, J. J.

Luca, F., see Faye, B.

Luca, F., see Kozek, M.

Lynch, R. \& Morton, P., The quartic Fermat equation in Hilbert class fields of imaginary quadratic fields

\section{M}

Ma, Z. S., see Cui, S.-P.

Mance, B., see Li, B.

Maples, K. \& Rodgers, B., Bootstrapped zero density estimates and a central limit theorem for the zeros of the zeta function

Marcolli, M., see Lo, C. W. K.

Massé, B. \& Schneider, D., Fast growing sequences of numbers and the first digit phenomenon

Mayer, D. C., see Azizi, A.

Mazumdar, E., see Adhikari, S. D.

McCarthy, D. \& Papanikolas, M. A., A finite field hypergeometric function associated to eigenvalues of a Siegel eigenform

Meher, J., see Das, S.

Mignot, T., Points de hauteur bornée sur les hypersurfaces lisses de l'espace triprojectif

Miyatani, K., Monomial deformations of certain hypersurfaces and two hypergeometric functions

Morton, P., see Lynch, R.

Moscariello, A., On integers which are representable as sums of large squares

Mu, Q., see Zhong, T.

7 (2015) 2065

Murru, N., On the periodic writing of cubic

irrationals and a generalization of Rédei functions

3 (2015) 779

3 (2015) 843

1 (2015) 185

5 (2015) 1691

5 (2015) 1633

6 (2015) 1961

7 (2015) 2151

2 (2015) 407

7 (2015) 2087

2 (2015) 311

3 (2015) 705

4 (2015) 1177

5 (2015) 1451

8 (2015) 2431

3 (2015) 835

3 (2015) 945

8 (2015) 2405

6 (2015) 1961

8 (2015) 2505

\section{N}

Nam, K. S., see Gil, B. K.

Nam, K. S., see Gil, B. K.

Nathanson, M. B., A forest of linear fractional transformations

Nowak, W. G., Scheicher, K. \& Sirvent, V. F., On Hausdorff dimension monotonicity of a family of dynamical subsets of Rauzy fractals

2 (2015) 613

3 (2015) 1061

4 (2015) 1275

4 (2015) 1089

\section{$\mathbf{P}$}

Papanikolas, M. A., see McCarthy, D.

Park, H. W., see Gil, B. K. Park, H. W., see Gil, B. K. Park, P.-S., see Gil, B. K. Park, P.-S., see Gil, B. K. Park, S., see Chua, L.

Parks, J., Amicable pairs and aliquot cycles on average

Parry, D., A polynomial variation of Meinardus' theorem

8 (2015) 2431

2 (2015) 613

3 (2015) 1061

2 (2015) 613

3 (2015) 1061

4 (2015) 1345

6 (2015) 1751

Pasten, H., The ABC conjecture, arithmetic progressions of primes and squarefree values of polynomials at prime arguments

3 (2015) 721

Patkowski, A. E., A strange partition theorem related to the second Atkin-Garvan moment

Pejković, T., see Bugeaud, Y.

1 (2015) 251

Pereira, W., see Birbrair, L.

2 (2015) 495

Pollack, P. \& Thompson, L., Arithmetic functions at consecutive shifted primes

5 (2015) 1477

5 (2015) 1633

Pollack, P., see Kozek, M.

Pomerance, C., see Freiberg, T. 
Pomerance, C., see Kozek, M.

Popa, D., see Bănescu, M.

Porta, H. A. \& Stolarsky,

K. B., A golden iterated map number system:

Results and conjectures

Pupyrev, Yu., On a lower bound for $\left\|(4 / 3)^{k}\right\|$

Q

Qian, G., see Qin, X.

Qin, X., Qian, G. \& Hong, S., New results on permutation polynomials over finite fields

$\mathbf{R}$

Roberts, D. P., Polynomials with prescribed bad primes

Rodgers, B., see Maples, K.

\section{$\mathbf{S}$}

Saikia, N., see Barman, R. Samuels, C. L. \& Strunk, T. J., Optimal factorizations of rational numbers using factorization trees

Sanna, C., On the number of arithmetic formulas

Saradha, N. \& Shorey, T. N., Squares in blocks from an arithmetic progression and Galois group of Laguerre polynomials

Sasaki, Y., Zeta Mahler measures, multiple zeta values and L-values

Saunders, J. C., Sums of digits in q-ary expansions

Scheicher, K., see Nowak, W. G.

Scheidler, R., see Jacobson, Jr., M. J.

Schneider, D., see Massé, B.

Schultz, D., Solving polynomial equations with elliptic modular functions

Schwagenscheidt, M. \& Völz, F., Lifting
5 (2015) 1633

7 (2015) 2073

5 (2015) 1617

1 (2015) 299

2 (2015) 437

2 (2015) 437

4 (2015) 1115

7 (2015) 2087

2 (2015) 645

3 (2015) 739

4 (2015) 1099

1 (2015) 233

7 (2015) 2239

2 (2015) 593

4 (2015) 1089

6 (2015) 1839

3 (2015) 705

4 (2015) 1313 newforms to

vector-valued modular

forms for the Weil

representation

7 (2015) 2199

Sedunova, A., On the asymptotic formulae for some multiplicative functions in short intervals

5 (2015) 1571

Sellers, J. A., see Chen, S.-C.

5 (2015) 1463

Shen, L. M., see Zhong, T.

7 (2015) 2065

Shorey, T. N., see Saradha, N.

1 (2015) 233

Şiar, Z., On square classes in generalized Lucas sequences

2 (2015) 661

8 (2015) 2325

Silas, S, see Chen, Y.

Sirvent, V. F., see Nowak, W. G.

4 (2015) 1089

Stolarsky, K. B., see Porta, H. A.

5 (2015) 1617

Straub, A., Congruences for Fishburn numbers modulo prime powers

5 (2015) 1679

Strunk, T. J., see Samuels, C. L.

3 (2015) 739

Sugiyama, R., Remark on nondegeneracy of simple abelian varieties with many endomorphisms

Sun, Q. F., On effective determination of symmetric-square lifts, level aspect

$1(2015) 51$

Sun, Z. H., Congruences for $q^{[p / 8]}(\bmod p)$ under the condition $4 n^{2} p=x^{2}+q y^{2}$

4 (2015) 1301

Sun, Z.-H., Super congruences concerning Bernoulli polynomials

8 (2015) 2393

Szalay, L. \& Ziegler, V., $S$-Diophantine quadruples with $S=\{2, q\}$

3 (2015) 849

Szczuka, P., The closures of arithmetic progressions in Kirch's topology on the set of positive integers

3 (2015) 673

$\mathbf{T}$

Takeshi, N., Elliptic curves with good reduction everywhere over cubic 
fields

Taous, Md., see Azizi, A.

Thompson, L., see

Pollack, P.

Thunder, J. L., see

Kettlestrings, D.

Tretkoff, P., K3 surfaces with algebraic period ratios have complex multiplication

Treviño, E., The Burgess inequality and the least $k$ th power non-residue

Trifonov, O., see Filaseta, M.

Tsaknias, P., see Freitas, N.

Tsukerman, E., Equality of Dedekind sums mod $\mathbb{Z}, 2 \mathbb{Z}$ and $4 \mathbb{Z}$

V

Väänänen, K., On rational approximations of certain Mahler functions with a connection to the Thue-Morse sequence

Vaughan, R. C., Squares: Additive questions and partitions

Völz, F., see

Schwagenscheidt, M.

\section{W}

Wang, L., Arithmetic properties of partition triples with odd parts distinct

Wang, S., Maximal height of divisors of $x^{p q^{b}}-1$

Wang, X. H., see Chen, $\mathrm{H}$. B.

Wei, B., Exponential sums twisted by Fourier coefficients of automorphic cusp forms for $\mathrm{SL}(2, \mathbb{Z})$

Weitzer, M., Characterization algorithms for shift radix systems with finiteness property

Wen, Z. X., see Chen, H. B.

Williams, H. C., see Jacobson, Jr., M. J.
1 (2015) 211

7 (2015) 2175

4 (2015) 1149

4 (2015) 1177

5 (2015) 1477

$2(2015) 569$

5 (2015) 1709

5 (2015) 1653

5 (2015) 1411

1 (2015) 81

6 (2015) 1735

$2(2015) 487$

5 (2015) 1367

7 (2015) 2199

6 (2015) 1791

1 (2015) 67

7 (2015) 2175

1 (2015) 39

6 (2015) 1839
Williams, K. S., see Evans,

R. J.

8 (2015) 2487

Wu, W., see Han, G.-N.

6 (2015) 1887

\section{Y}

Yamamoto, S., A sum formula of multiple $L$-values

1 (2015) 127

Yang, H. \& Fu, R., An upper bound for least solutions of the exponential Diophantine equation

$D_{1} x^{2}-D_{2} y^{2}=\lambda k^{z}$

4 (2015) 1107

Yao, O. X. M., Arithmetic properties for Fu's 9 dots bracelet partitions

Yasuda, M., Ramification of the Kummer extension generated from torsion points of elliptic curves

6 (2015) 1725

Yazdani, S., see Bennett, M. A.

1 (2015) 1

Ye, D., Evaluation of the convolution sums $\sum_{l+36 m=n} \sigma(l) \sigma(m)$ and
$\sum_{4 l+9 m=n} \sigma(l) \sigma(m)$

1 (2015) 171

Ye, D., Representations of certain binary quadratic forms as a sum of Lambert series and eta-quotients

Yuan, A., see Chua, L.

4 (2015) 1073

4 (2015) 1345

\section{$\mathbf{Z}$}

Zábrádi, G., see

Backhausz, T.

4 (2015) 1233

Zekhnini, A., see Azizi, A.

4 (2015) 1177

Zeng, J., see Liu, Z.-G.

2 (2015) 507

Zhang, W.-B. (Cheung, M. P.), Extensions of Beurling's prime number theorem

5 (2015) 1589

Zhang, Y., see Cai, T.

Zhang, Z.-L., On the exceptional sets in Sylvester continued fraction expansion

Zhao, J., see Guo, L.

1 (2015) 159

Zhao, P. P., see Gao, W. D.

8 (2015) 2369

3 (2015) 997

7 (2015) 2141

Zhelezov, D., Improved bounds for arithmetic 
progressions in product sets

Zhong, T., Mu, Q. \& Shen, L. M., Metrical property for $G C F_{\epsilon}$ expansion with

\begin{tabular}{|c|c|c|}
\hline \multirow[t]{3}{*}{$8(2015) 2295$} & $\begin{array}{l}\text { the parameter function } \\
\epsilon(k)=c(k+1)\end{array}$ & 7 (2015) 2065 \\
\hline & $\begin{array}{l}\text { Zhuang, J. J., see Gao, } \\
\text { W. D. }\end{array}$ & 7 (2015) 2141 \\
\hline & Ziegler, V., see Szalay, L. & $3(2015) 849$ \\
\hline
\end{tabular}

